\title{
Ensemble of Classifiers Based on Hard Instances
}

\author{
Isis Bonet, Abdel Rodríguez, Ricardo Grau, and María M. García \\ Center of Studies on Informatics, Central University of Las Villas, Santa Clara, Cuba \\ \{isisb, abdelr, rgrau, mmgarcia\}@uclv.edu.cu
}

\begin{abstract}
There are several classification problems, which are difficult to solve using a single classifier because of the complexity of the decision boundary. Whereas, a wide variety of multiple classifier systems have been built with the purpose of improving the recognition process. There is no universal method performing the best. The aim of this paper is to show another model of combining classifiers. This model is based on the use of different classifier models. It makes clusters to divide the dataset, taking into account the performance of the base classifiers. The system learns how to decide from the groups, by a meta-classifier, who are the best classifiers for a given pattern. In order to compare the new model with well-known classifier ensembles, we carried out experiments with some international databases. The results demonstrate that this new model can achieve similar or better performance than the classic ensembles.
\end{abstract}

Keywords: multiple classifiers, ensemble classifiers, classification, pattern recognition.

\section{Introduction}

Supervised learning is a common task in pattern recognition and machine learning, but not completely solved. A lot of classification methods have been built, however, none of them have proven to perform the best. In fact, a research area has been created to develop methods for combining classifiers, which goal is to fuse different classifiers, hoping that the performance reached will be better than the single classifiers.

There are some reasons to justify the use of classifier ensembles, but the more general justification is when we are dealing with a dataset which decision boundary is too complex to be learned by a single classifier [1]. This also seems to happen in too large or too short data sets. A database with these characteristics is also sensitive to expect low performance levels with a single classifier. Multiple classifier systems precisely arise from these problems in several tasks to combine different classification models. Ideally, it should choose the appropriate base classifier for each case of the dataset.

The design of classifier ensembles can be divided in three important steps. The first one is the definition of the topology: the distribution and connection of components. The second step, very related with the first one, is the choice of the base classifiers, 
which could be the key of success depending on the classifier diversity, a subject still open to research. The last step is the combination of classifier decisions, divided in two main strategies: selection and fusion.

There is a wide variety of these methods reported in the literature; indeed, some authors have made a good review and comparison of them [1], [2], [3], [4]. Even though there exist many classifier ensembles, the most popular ensembles are: Bagging [5], Boosting [6] and Stacking [7].

Bagging and Boosting are based on the manipulation of the training base to achieve the diversity of base classifiers. The diversity is obtained by using bootstrapped replicas of the training data. All classifiers are instances of the same model and the output combination is obtained by the majority vote.

Boosting is similar to Bagging, but once a classifier is trained, it uses its mistakes to weight the samples for the training set of the next classifier, reinforcing misclassified samples. AdaBoost [8] is a more general version of this algorithm and actually more known than Boosting. In this paper when we refer to Boosting we are actually talking about this version: AdaBoost.

On the other hand, Stacking uses different classification models with the same training sets and has a second classifier level provided for a meta-classifier that works as the outputs combiner. This combiner is trained on a data set obtained from the relation between the classifiers outputs and the real value of the objective feature for each pattern.

In short, we can divide the classifier ensembles according to if they use the same classifier model and only change the parameters for training: datasets, features, model parameters; or if they use different classifier models. Bagging and Boosting are examples of using the same model, whereas Stacking is an example of the second type of ensembles.

Despite the classifier ensembles have been successfully applied in complex realword pattern recognition problems, some complex real-problems are not solved and the searching for new models of combined classifiers is still an open research challenge.

The aim of this paper is to present a new way of combining classifiers based on the ensemble with different classifier models. The research is focused on the topology of the model and the learning process, not on how to guarantee diversity. In order to test the presented model we carried out experiments on 34 data sets and also a comparison is shown with some popular classifier ensembles.

\section{Ensemble of Classifiers Using a Meta-classifier by Clusters of Hard Instances}

The proposed model in this paper is based on the philosophy of stacking. It uses different classification models and a meta-classifier to combine the decisions, as Stacking does. Moreover the training data to the meta-classifier is built from the outputs of the base classifiers. The principal difference is on the way to train the meta-classifier. In stacking the original representation of patterns changes, now the features are the classifiers, where the values are the outputs of them, keeping the same objective feature. On the other hand, the proposed model also build a database to train 
the meta-classifier, but the patterns keep the same features of the original data and the objective feature now represent the classifier that provides good classification for the pattern.

In order to give an insight into the workings of the proposed model its different parts will be defined below.

\subsection{Topology}

The general objective function is defined for classification problems (equation 1).

$$
\text { FO: } R D \rightarrow O
$$

Where $R D$ is the features set and $O=\{1,2, \ldots, N\}$ is the set of $N$ possible values of the objective feature (classes).

For a given classification problem the use of different classifier models can provide different decision boundaries. Hence, the adequate selection of classifiers is the key for the success [9].

Once the selection of potential classifiers is done, the question is: "Which of them are the best for classifying a given pattern?" That process can be very complex because each classifier can define a different decision boundary to separate the classes. This can result in different outputs from the classifiers for a given instance. What classifier should we select? Ideally, we know what each classifier has learned. To take into account this idea, the model developed in this work is based on the correct classified patterns for each classifier, and the misclassified patterns will be dismissed, they will produce an error anyway.

Now the problem is to define the method for combining the outputs, but in this model this is also related with the design of the topology. In order to do it, we consider the best classifiers of the ensemble for a given pattern by associating them with the group of correctly classified patterns. Once the classifiers are trained, each one will have a group of correct patterns associated, that we will call "hard instances". An output combination could be useful to find what patterns each classifier has learned. Afterwards, we could separate the data instances in clusters according to the classifier outputs; suppose we have $C$ base classifiers, so we would be in presence of $C$ clusters.

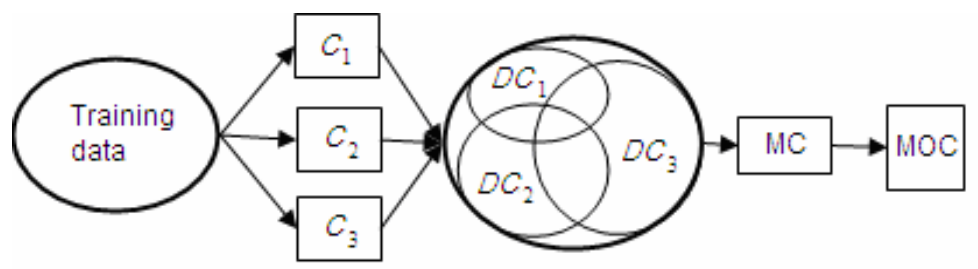

Fig. 1. Example of the multiclassifier using three base classifier models

Figure 1 depicts an example of the proposed model using $C=3$ base classifiers, where classifiers $C_{1}, C_{2}$, and $C_{3}$ are trained from the training data. Afterwards, as it can be seen in the figure, the result can be interpreted as a clustering process of the 
dataset. Each classifier will have a subset of hard instances, these are represented by circle inside the data $\left(D C_{1}, D C_{2}, D C_{3}\right)$, to give a better understanding of the problem. Usually these subsets have common patterns.

The figure shows a subset of patterns which are out of the three clusters; these are misclassified by all classifiers. There is no way to combine the base classifiers in order to obtain another result for the last set of misclassified patterns. Hence, those patterns can be excluded of the training data for the meta-classifier.

This system is based on adding a meta-classifier that learns how to separate the patterns according to the $C$ groups. In many data sets we will have a high significance percentage of patterns that can be easily classified by any classifier, and in this case, we would have this patterns repeated in all $C$ clusters, that means that the region in the interception of all clusters of hard instances can be too big. This is another patterns subset to be taken into account. In the figure we can see it in the interception of the three circles; this represents the patterns that are correctly classified by all classifiers in the model. Then we create a $C+1$ cluster with the patterns in the interception of all previous ones in order to reduce the noise caused by repeated patterns in all groups. The creation of this new group does not eliminate all repeated cases associated to different classes because we also have the interception of clusters by pairs, but these repeated instances should not be so significant. Anyway, it is important to keep in mind selecting a meta-classifier that can use a continuous valued output, in order to use the probability associated to every class (in this case the class represents the classifiers) as the measure to combine the outputs of the different classifiers.

In short, in order to balance the classes in the new database, we select a threshold to decide when the case is kept in the $C+1$ group or added to any other group. Usually, when the classifiers produce an outputs' vector, a threshold is establish to decide what the resulting class is. In the case of two classes this threshold is 0.5 by default. The variety of this threshold can drastically change our clusters, but also can redistribute better the patterns in the different sets. Of course, this threshold depends on the base, for this reason it must be considered as a parameter of the model.

We build a new dataset from the formed clusters of hard instances to train the metaclassifier. The difference between the objective function for the base classifier and the meta-classifier is given by the objective feature. Given a problem which training data has $N$ possible values the objective feature is $O=\{1,2, \ldots, N\}$ for the base classifiers. The objective feature for the meta-classifier is $O=\{1,2, \ldots, C+1\}$. Notice that now the objective feature is composed by values corresponding with $C$ classifiers and an extra $C+1$ that is the subset of patterns that are correctly classified by all base classifiers.

We can find some problems in the new database, created for the meta-classifier. A classifier has a small cluster of correct classified instances, resulting in unbalanced groups. Sometimes that problem can be solved selecting other base classifiers or changing the threshold value.

Another problem is found when a large number of examples are misclassified by all base classifiers. In that case, the meta-classifier does not have any possibility to change the results, for this reason these cases are not important for our model. 
Some authors have developed many diversity measures that help us to select base classifiers[10], [11], [12]. Nevertheless, in this paper we do not pretend to optimize the search of the classifiers, just to propose a different paradigm, as a different way for finding the decision regions.

Ideally, for an specific data we may select the appropriate classifiers using any diversity measure [10].

\subsection{Combining Outputs}

In this work we propose two ways to combine the outputs, one based on selection and another based on fusion.

The combination by selection is the simplest, consisting on the selection of the classifier with the bigger probability, taking into account the outputs vector of metaclassifier. It can also be seen as an alternative approach to the classifier combination, called dynamic classifier selection [13].

The fusion combination is based on weighting the outputs of the classifiers with the output of the meta-classifier. The meta-classifier is trained with this new database, explained in the topic 2.1 , so it will have a vector of probabilities associated to the clusters as output. Then, the module of outputs combination (MOC) takes it to weigh the classifiers, as it is shown in the equation 2.

$$
\mathrm{S}=\mathrm{SC} * \mathrm{SMC}
$$

Where, $S C$ is a matrix $C \mathrm{x} N, C$ is the number of classifiers and $N$ the amount of classes. $S C$ is the matrix of classifier outputs, that is, $S C(i, j)$ is the probability given by the classifier $i$ for class $j$. SMC is the probabilities' vector given by the metaclassifier. $S$ is the vector of probabilities, with dimension $N$, which results from the system.

The proposed method will be called "Multi-Expert by Hard Instances" (MEHI). It was implemented by using Weka (created in the University of Weikato in New Zealand) [14]. This software offers a great number of classification methods and facilities to add new models and to compare with others.

\section{Result and Discussion}

To validate this new model, 34 bases of general classification problems were chosen from the UCI Repository [15]. The databases are miscellaneous, 10 of them have discrete features, 11 have continuous features and 13 have mixed features. With respect to the objective feature they also present diversity, the amount of value ranges is between 2 and 24. Also the amount of samples varies between 23 and 3772 .

Several topologies were used in this work to choose the base classifiers and the meta-classifier. Finally, 3 base classifiers were selected: decision tree (J48), Bayesian Network (BayesNet) and Support Vector Machine (SVM). Multilayer Perceptron (MLP) was used as meta-classifier. A study of the threshold was made to build the clusters by classifiers in correspondence with each base, these thresholds oscillated between 0.6 and 0.9 . We used 10 -fold cross-validation, in order to validate the results and to compare them with other methods. 

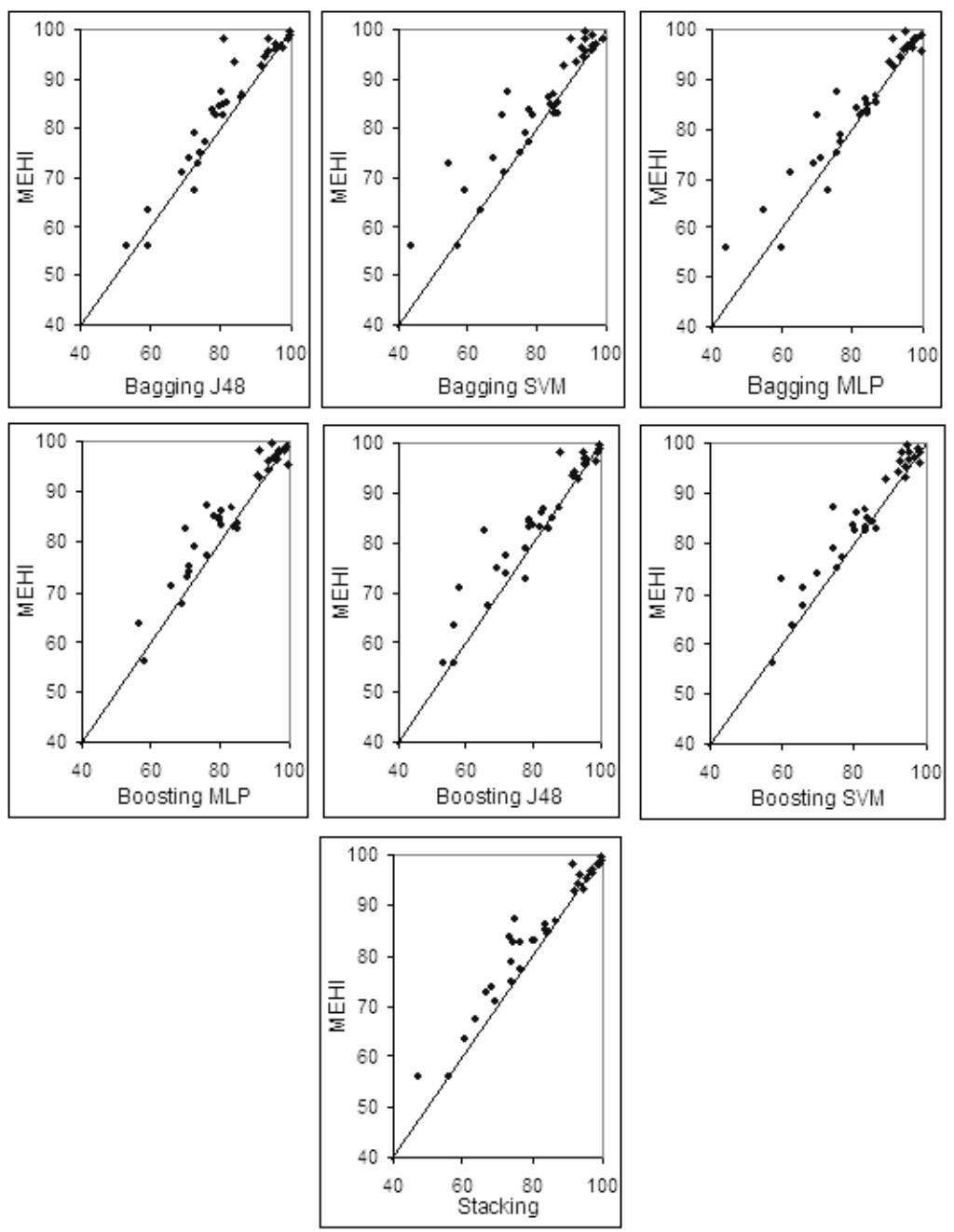

Fig. 2. Comparison of the results from the 11 databases using Bagging, Boosting, Stacking and the new model: MEHI

To validate the new method, Bagging, AdaBoost and Stacking were chosen. In the case of Bagging and AdaBoost they were tested with three base classifiers: J48, SVM and MLP. Bayesian Network was not used because of the stability of this method. On the other hand, Stacking was tested with the same classifier models used in the proposed method.

The results are shown in figure 2 based on the accuracy of each method. Each graph represents the comparison between MEHI and each of the ensembles selected: Bagging, Boosting and Stacking. Each dot represents a database. If the point lies above the line means that MEHI achieved higher accuracy than another ensemble. 
The three graphs in the top of the figure show the results of MEHI versus Bagging with different models. The first one is with Bagging using J48 as base classifier, the second one using SVM and the last one using MLP. In the middle of the figure the results of Boosting are shown in the same way. In both cases the results are similar; the proposed model is superior in most of the databases. But, it is also true that many of them are on the line or close to it, this means that Bagging and Boosting can be better for some databases.

The comparison with Stacking is in the bottom of the figure, showing again that MEHI has better results.

Both ways of combination: selection and fusion were used in the experiments. The results shown in figure 2 are related with de combination by fusion. The performance obtained using combination by selection, is similar, although a little lower, than the combination by fusion shown above. For this reason we select combination by fusion to show in this experiment.

Although we do not intend to propose our method as the best ensemble for classification database, we suggest it as an alternative model to be taken into account when the ensembles are needed to solve problems of complex decision boundaries.

In general, the results show that in most of the bases the new model are superior or at least similar to those obtained by the other classifier ensembles.

A statistical study with Wilcoxon signed rank test could conclude that the differences in performance are significant in all cases, with MEHI ahead.

\section{Conclusions}

In this work, a model of combination of classifiers based on their local performance was designed and implemented. This result was obtained by a meta-classifier that learns how to separate the correctly classified instances by each base model. This method was compared with well-known classifier ensembles and its accuracy was significantly superior for a sample of 34 international databases of UCI Repository. Therefore, we conclude that the proposed classifier ensemble improves the results of accuracy and should be tested in real-world databases. It is important to remark that the methods do not replace none other ensemble, it is just another way to combine multiple classifiers.

\section{References}

1. Polikar, R.: Ensemble based systems in decision making. IEEE Circuits and Systems Magazine 6, 21-44 (2006)

2. Dietterich, T.G.: Ensemble methods in machine learning. In: Kittler, J., Roli, F. (eds.) MCS 2000. LNCS, vol. 1857, pp. 1-15. Springer, Heidelberg (2000)

3. Ghosh, J.: Multiclassifier systems: Back to the future. In: Roli, F., Kittler, J. (eds.) MCS 2002. LNCS, vol. 2364, pp. 1-15. Springer, Heidelberg (2002)

4. Kuncheva, L.I.: Combining Pattern Classifiers, Methods and Algorithms. Wiley Interscience, New York (2004)

5. Breiman, L.: Bagging predictors. Machine Learning 24, 123-140 (1996)

6. Schapire, R.E.: The strength of weak learnability. Machine Learning 5, 197-227 (1990) 
7. Wolpert, D.: Stacked generalization. Neural Networks 5, 241-259 (1992)

8. Freund, Y., Schapire, R.E.: Decision-theoretic generalization of on-line learning and an application to boosting. Journal of Computer and System Sciences 55, 119-139 (1997)

9. Canuto, A.M.P., Abreu, M.C.C., Oliveira, L.D., Xavier, J.C., Santos, A.D.: Investigating the influence of the choice of the ensemble members in accuracy and diversity of selection-based and fusion-based methods for ensembles. Pattern Recognition Letters 28, 472-486 (2007)

10. Kuncheva, L.I., Whitaker, C.J.: Measures of diversity in classifier ensembles and their relationship with the ensemble accuracy. Machine Learning 51, 181-207 (2003)

11. Banfield, R.E., Hall, L.O., Bowyer, K.W., Kegelmeyer, W.P.: Ensemble diversity measures and their application to thinning. Information Fusion 6, 49-62 (2005)

12. Tang, E.K., Suganthan, P.N., Yao, X.: An analysis of diversity measures. Machine Learning 65, 247-271 (2006)

13. Giacinto, G., Roli., F.: Dynamic classifier selection. In: 1st International Workshop on Multiple Classier Systems, pp. 177-189 (Year)

14. Witten, I., Frank, E.: Data Mining: Practical Machine Learning Tools and Techniques. Diane Cerra, San Francisco (2005)

15. University of California, Irvine, School of Information and Computer Sciences, http: //www.ics.uci.edu/ mlearn/MLRepository.html 